\title{
Nighttime Symptoms After Monocular SMILE: A Contralateral Eye Study
}

\author{
Wuxiao Zhao · Tian Han · Meiyan Li · Walter Sekundo • \\ Aruma Aruma $\cdot$ Xingtao Zhou it
}

Received: August 4, 2021 / Accepted: September 6, 2021 / Published online: September 24, 2021

(C) The Author(s) 2021

\begin{abstract}
Introduction: To investigate nighttime symptoms in patients with myopic anisometropia after monocular small incision lenticule extraction (SMILE) surgery.

Methods: Thirty-six patients who had undergone monocular SMILE more than 6 months previously were recruited at the Eye \& ENT Hospital of Fudan University. The average age at surgery was $25.4 \pm 6.1$ years. Preoperative
\end{abstract}

Wuxiao Zhao and Tian Han contributed equally to this study.

W. Zhao · T. Han $\cdot$ M. Li · A. Aruma $\cdot$ X. Zhou ( $\square)$ Eye Institute and Department of Ophthalmology and Optometry, Eye \& ENT Hospital of Fudan University, No.19 Baoqing Road, Shanghai 200031, China

e-mail: doctzhouxingtao@163.com

W. Zhao $\cdot$ T. Han $\cdot$ M. Li · A. Aruma $\cdot$ X. Zhou NHC Key Laboratory of Myopia (Fudan University), Shanghai, China

W. Zhao $\cdot$ T. Han $\cdot$ M. Li · A. Aruma $\cdot$ X. Zhou Shanghai Research Center of Ophthalmology and Optometry, Shanghai, China

W. Zhao - T. Han - M. Li - A. Aruma - X. Zhou Shanghai Engineering Research Center of Laser and Autostereoscopic 3D for Vision Care (20DZ2255000), Shanghai, China

W. Sekundo

Department of Ophthalmology, Phillips University

of Marburg, Marburg, Germany spherical equivalent (SE) was $-3.77 \pm 1.56 \mathrm{D}$ in SMILE-treated eyes and $-0.08 \pm 0.66 \mathrm{D}$ in unoperated eyes. Main measurements included uncorrected and corrected distance visual acuity, manifest refraction, halo radius, contrast sensitivity, nighttime symptoms, and patient satisfaction.

Results: The mean follow-up time was $13.9 \pm 3.4$ months. The efficacy and safety indexes were 1.18 and 1.28 , respectively. The halo radius was not significantly different between SMILE-treated and unoperated eyes under luminance conditions of 1,5 , and $100 \mathrm{~cd} /$ $\mathrm{m}^{2}(P=0.055)$. No significant differences were observed in contrast sensitivity at all spatial frequencies between eyes under both uncorrected and corrected conditions (all $P>0.05$ ). None of the patients reported moderate or severe symptoms at night. Mild symptoms (glare, halo, starburst) were reported and binocularly equal in 13 patients, whereas four patients reported better night vision in SMILE-treated eyes than unoperated eyes, and one of them experienced mild night vision disturbance. The overall satisfaction score was $9.39 \pm 0.80$.

Conclusions: The disk halo size and contrast sensitivity in SMILE-treated eyes were similar to those in unoperated eyes, and nighttime symptoms almost completely resolved after SMILE. 
Keywords: Contrast sensitivity; Disk halo size; Nighttime symptom; Small incision lenticule extraction

\section{Key Summary Points}

\section{Why carry out this study?}

Nighttime symptoms, especially glare and halo, are common complications of small incision lenticule extraction (SMILE). This study evaluated refractive outcomes, disk halo size, contrast sensitivity, and nighttime symptoms in monocular SMILE patients.

Thirty-six patients who had undergone monocular SMILE more than 6 months previously were recruited, and visual performance was measured by the MonPack One (Metrovision, France); nighttime symptoms were assessed by patient-reported questionnaire on the basis of individual eyes.

\section{What was learned from this study?}

The efficacy index and safety index were $1.18 \pm 0.17$ and $1.28 \pm 0.18$ in SMILEtreated eyes, respectively.

Similar contrast sensitivity and high satisfaction scores were achieved in patients with myopic anisometropia after monocular SMILE surgery.

Nighttime symptoms (glare, halo, starburst) became mild or disappeared for most individuals over 14 months postoperatively.

Patients reported interocular visual differences indicating that night vision in SMILE-treated eyes was superior to that in unoperated eyes.

Monocular SMILE surgery contributed to the recovery of normal vision in specific myopic anisometropic patients, but the nomogram should be considered to avoid myopic shift occurring in unoperated eyes.

\section{INTRODUCTION}

Small incision lenticule extraction (SMILE) is a corneal refractive procedure for myopia and myopic astigmatism correction with great predictability and stability $[1,2]$. Visual acuity and vision quality are important for refractive surgeries. Previous studies have documented excellent visual quality after SMILE using subjective or objective measurements, such as the OQAS (Optical Quality Analysis System) [3], C-Quant straylight meter [4], and subjective questionnaires [5-9]; other vision quality measurements including objective scatter index, contrast sensitivity, and uncorrected visual acuity returned to preoperative levels after 3 months $[3,6,8,9]$. Our team also observed that the disk halo size, which is a veiling light over the retina produced by the forward-scattered light into the eye and which induces a disability glare [10-12], temporarily increased and then returned to baseline 3 months after SMILE [13]. However, some patients still encountered glare when driving at night or recognizing a target against a bright background even 3 years after the surgery [5]. Surgery-induced night symptoms are worth discussing, and a self-controlled study design is a common and valid way to explore such practical questions. However, comparing the symptoms between the operated and unoperated eyes of the same patient can provide real-time information and remove the potential bias of individual characteristics [14].

To our knowledge, no study has focused on such a special kind of myopic anisometropia. This study investigated disk halo size, contrast sensitivity, and nighttime symptoms in patients after monocular SMILE surgery.

\section{METHODS}

\section{Patients}

Thirty-six patients (18 men and 18 women) who had undergone monocular SMILE (27 right eyes and 9 left eyes) more than 6 months previously were recruited in this retrospective study. The surgical procedures were conducted 
in the Eye, Ear, Nose, and Throat (EENT) Hospital of Fudan University from February 2016 to March 2020. The average age at surgery was $25.4 \pm 6.1$ years. The preoperative spherical equivalent (SE) was $-3.77 \pm 1.56 \mathrm{D}$ in the SMILE-treated eyes and $-0.08 \pm 0.66 \mathrm{D}$ in the unoperated eyes (Table 1). Among SMILE-treated eyes, 29 were dominant eyes.

The preoperative inclusion criteria were as follows: age greater than 18 years, SE changes within 2 years less than or equal to $-1.00 \mathrm{D}$, corrected distance visual acuity (CDVA) of $20 / 25$ or better, and no use of soft contact lenses for more than 2 weeks, hard contact lenses for more than 1 month, or ortho-K contact lenses for more than 3 months.

This study complied with the principles of the Declaration of Helsinki and was approved by the Ethics Committee of the EENT Hospital of Fudan University. A consent form for participation and academic publication request was obtained from each patient.

\section{Surgical Technique}

All surgeries were performed by a single experienced surgeon (XZ). A VisuMax femtosecond laser (Carl Zeiss Meditec AG) was used, with a frequency of $500 \mathrm{~Hz}$ and pulse energy of $130 \mathrm{~nJ}$. The lenticule diameter was set between $6.6 \mathrm{~mm}$ and $7.0 \mathrm{~mm}$; the cap diameter was set to
$7.5 \mathrm{~mm}$ at $120-\mu \mathrm{m}$ depth in a $90^{\circ}$ single-side cut, with a length of $2.0 \mathrm{~mm}$.

The postoperative prescription was as follows: levofloxacin eye drops four times per day for 7 days; $0.1 \%$ fluorometholone eye drops eight times per day, reduced by one time every 3 days for a total of 24 days; and preservativefree artificial tears four times per day for 1 to 2 months.

\section{Disk Halo Size Measurement}

The halo radius was measured using a vision monitor (MonPack One, Metrovision, France) in a dark room. As described previously [15], patients with the best spectacle correction were tested monocularly at a distance of $2.5 \mathrm{~m}$ after 5 min of adaptation to darkness. The test was performed using letters with luminance conditions of 1,5 , and $100 \mathrm{~cd} / \mathrm{m}^{2}$. The light source positioned to the right of the patient was used to test the right eye, and that positioned to the left of the patient was used to test the left eye. Three radial lines with 10 letters, forming 10 rings at intervals of 30 arc min, were displayed from the periphery toward the light source. To avoid a retinal afterimage, patients were instructed to adopt an out-to-in strategy to read the letters on each line from the side opposite the light source. The average of unrecognized letters in the three lines was calculated as the halo radius in arc min.

Table 1 Preoperative demographics and refraction data

\begin{tabular}{llll}
\hline & SMILE-treated eyes & Unoperated eyes & $p$-value \\
\hline Age, year & $25.4 \pm 6.1$ & & - \\
Sex (male/female) & $18 / 18$ & & - \\
Sphere, diopters & $-3.49 \pm 1.55$ & $-0.22 \pm 0.62$ & 0.000 \\
Range, diopters & -6.25 to -0.75 & -0.75 to +1.75 & \\
Cylinder, diopters & $-0.56 \pm 0.42$ & $-0.59 \pm 0.48$ & 0.720 \\
Range, diopters & -1.50 to 0 & -1.75 to +0.47 & \\
SE, diopters & $-3.77 \pm 1.56$ & $-0.08 \pm 0.66$ & 0.000 \\
CDVA, logMAR & $-0.05 \pm 0.06$ & $-0.04 \pm 0.08$ & 0.103 \\
\hline
\end{tabular}

SE, spherical equivalent; CDVA, corrected distance visual acuity; logMAR, logarithm of the minimum angle of resolution 
Table 2 Patient questionnaire

\section{Parameter}

1 Do you experience any night vision disturbance currently? If YES, which eye?

$\square$ No $\square$ Yes (operated eye, unoperated eye, or both eyes)

2 During the last week, have you experienced interocular visual differences at night? If YES, which eye is better in term of night vision?

No $\square$ Yes (operated eye or unoperated eye)

3 During the last week, have you experienced glare at night? If so, which eye? And rate it as mild, moderate, or severe.

$\square$ No $\square$ Yes (operated eye, unoperated eye, or both eyes) $\square$ Mild $\square$ Moderate $\square$ Severe

4 During the last week, have you experienced halos (rings around lights) at night? If so, which eye? And rate it as mild, moderate, or severe.

$\square$ No $\square$ Yes (operated eye, unoperated eye, or both eyes) $\square$ Mild $\square$ Moderate $\square$ Severe

5 During the last week, have you experienced starburst around lights at night? If so, which eye? And rate it as mild, moderate, or severe.

No $\square$ Yes (operated eye, unoperated eye, or both eyes) $\square$ Mild $\square$ Moderate $\square$ Severe

6 During the last week, have you experienced any visual distortion as you normally function at night? If so, which eye? And rate it as mild, moderate, or severe.

$\square$ No $\square$ Yes (operated eye, unoperated eye, or both eyes) $\square$ Mild $\square$ Moderate $\square$ Severe

7 The overall satisfaction score (on a scale of $1-10,1=$ low satisfaction and $10=$ high) with your refractive surgery outcomes is

Mild: symptoms noted to affect light sources, but functions were not interfered; Moderate: symptoms noted and usual activity were affected, especially when driving or looking at light sources; Severe: certain activities, such as driving or looking at light sources, were restrained by symptoms

\section{Contrast Sensitivity Tests}

The contrast sensitivity was tested using the same vision monitor. The testing protocol has been described in detail previously [16]. The test was performed monocularly at a distance of $2 \mathrm{~m}$ under corrected and uncorrected conditions after adapting to darkness for $5 \mathrm{~min}$. Vertical gratings of sine waves of various spatial frequencies (SFs), including $0.5\left(\mathrm{SF}_{0.5}\right), 1.1\left(\mathrm{SF}_{1.1}\right)$, $2.2\left(\mathrm{SF}_{2.2}\right), 3.4\left(\mathrm{SF}_{3.4}\right), 7.1\left(\mathrm{SF}_{7.1}\right)$, and 14.6 $\left(\mathrm{SF}_{14.6}\right)$ cycles/degree (cpd), were displayed on the visual monitor in full-screen mode. The contrast gradually increased until the patient's response was received, and the results were recorded. Thereafter, the monitor presented a graph of the contrast sensitivity function and registered the contrast at each SF in $\mathrm{dB}$.

\section{Nighttime Symptoms and Patient Satisfaction}

Current nighttime symptoms, including glare, halo, starburst, distortion, and night vision disturbance (on a scale of none, mild, moderate, or severe), together with interocular differences regarding night vision were evaluated by a written questionnaire for each patient at the last follow-up. The above questions were solicited based on individual eyes rather than as a binocular assessment involving both eyes, and patient overall satisfaction scores (on a scale of $1-10$ points, $1=$ low, and $10=$ high) with their 
SMILE surgery outcomes were also recorded (Table 2).

\section{Statistical Analysis}

For $\alpha=0.05$ and $80 \%$ power, the null hypothesis mean (halo radius, $\mu_{0}$ ) was 88.4 arc min at $5 \mathrm{~cd} / \mathrm{m}^{2}$ level, the standard deviation $(\sigma)$ was 22.1 , and the true mean (halo radius, $\mu$ ) was 77.0 arc $\mathrm{min}$, for sample size calculation. The calculated number of eyes necessary for detecting differences in the halo radius was 32 (G*Power 3.1). IBM SPSS Statistics for Windows software (version 24, IBM Corp., Armonk, NY) was used for the statistical analyses in this study. The Shapiro-Wilk test was performed to assess the normality of contrast sensitivity and halo radius before the application of nonparametric tests. The differences in contrast sensitivity at different SFs, the halo radius at different luminance levels between SMILE-treated and unoperated eyes, and the intergroup differences in contrast sensitivity were analyzed by repeated-measures analysis of variance (ANOVA). Significance was set at $P<0.05$.

\section{RESULTS}

All patients underwent SMILE without any severe complications, such as corneal infection or diffuse lamellar keratitis. The average followup time was $13.9 \pm 3.4$ months (8-20 months).

\section{Efficacy, Safety, Predictability, and Astigmatism Correction}

The cumulative percentage of uncorrected distance visual acuity (UDVA) at postoperative 14 months is presented in Fig. 1A. The efficacy index (postoperative UDVA/preoperative CDVA) of the procedure was $1.18 \pm 0.17$. All SMILE-treated eyes had a postoperative UDVA of $20 / 20$ or better; $80.6 \%(29 / 36)$ of the SMILEtreated eyes gained one or more lines of CDVA. No SMILE-treated eyes lost one or more lines of CDVA (Fig. 1B), and the safety index (postoperative CDVA/preoperative CDVA) was $1.28 \pm 0.18$.
A scatter plot of the attempted versus achieved SE correction is shown in Fig. 1C. The SE in SMILE-treated eyes was $0.22 \pm 0.39 \mathrm{D}$, whereas that in unoperated eyes was $-0.30 \pm 0.64 \mathrm{D} ; 86.1 \%(31 / 36)$ of the SMILEtreated eyes were within $\pm 0.50 \mathrm{D}$, whereas $97.2 \%(35 / 36)$ were within $\pm 1.00 \mathrm{D}$ (Fig. 1D).

Refractive astigmatism is shown in Fig. 1E. The mean refractive astigmatism was $-0.20 \pm 0.18 \mathrm{D}$ (range, -0.50 to $0 \mathrm{D}$ ) in SMILEtreated eyes, and $100 \%$ of the SMILE-treated eyes were within $\pm 0.50 \mathrm{D}$.

\section{Disk Halo Size}

For letter luminance levels of 1,5 , and $100 \mathrm{~cd} /$ $\mathrm{m}^{2}$, the mean halo radius in the SMILE-treated eyes was $207.8 \pm 57.9$ arc $\min , 76.1 \pm 25.0$ arc $\mathrm{min}$, and $60.0 \pm 0.0$ arc $\mathrm{min}$, respectively, whereas that in the unoperated eyes was $219.4 \pm 48.2$ arc min, $80.8 \pm 26.9$ arc min, and $60.0 \pm 0.0$ arc min, respectively. The interaction effect between surgery and luminance level was not significant (Mauchly's test of sphericity with Greenhouse-Geisser correction, $F=2.490$, $P=0.113)$. The test of within-subject effects showed that the main effect of surgery on the halo radius was not significant between the SMILE-treated and unoperated eyes $(F=3.925$, $P=0.055)$. Moreover, the main effect of luminance level on halo radius was significant $(F=345.732, P<0.001)$, and pairwise comparison showed significant differences in halo radius between any two luminance levels in both SMILE-treated and unoperated eyes (all $P<0.001$; Fig. 2).

\section{Contrast Sensitivity}

Although the contrast values at all SFs were significantly different in each group (Table 3), a marginal but nonsignificant increase in contrast was observed in the SMILE-treated eyes compared to the unoperated eyes under uncorrected conditions (repeated-measures ANOVA, $F=2.027, P=0.163)$. No significant difference was noted under corrected conditions (repeated-measures ANOVA, $F=0.663, P=0.421$; Fig. 3). 


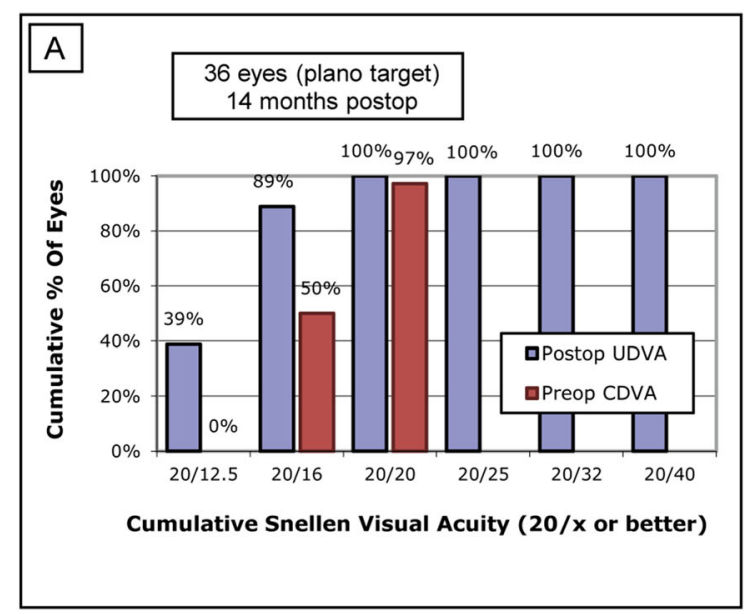

Uncorrected Distance Visual Acuity

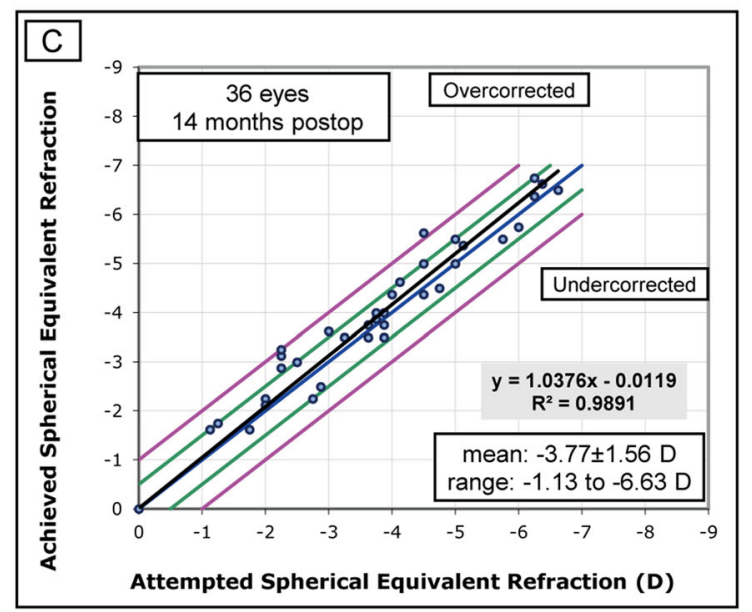

Spherical Equivalent Attempted vs Achieved

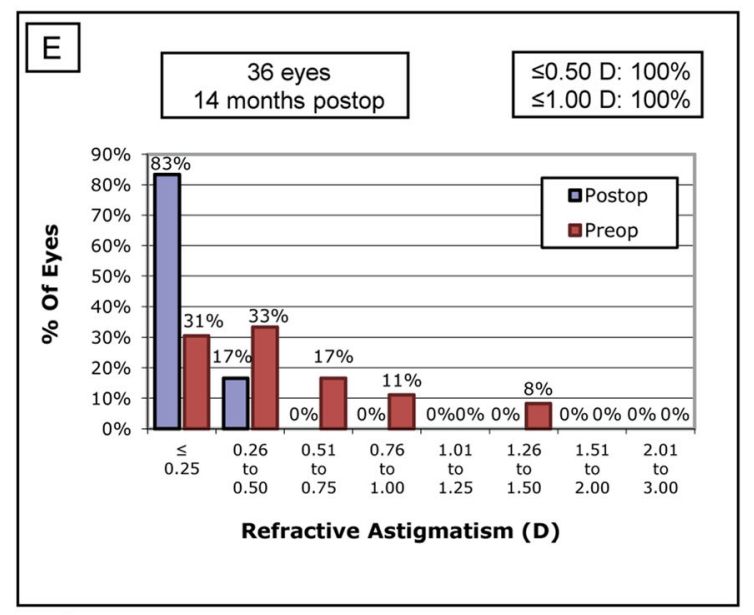

Refractive Astigmatism

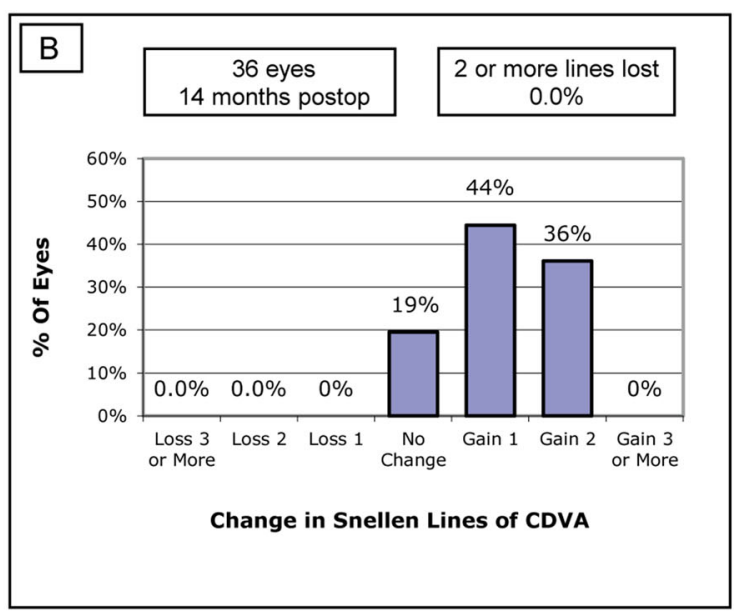

Change in Corrected Distance Visual Acuity

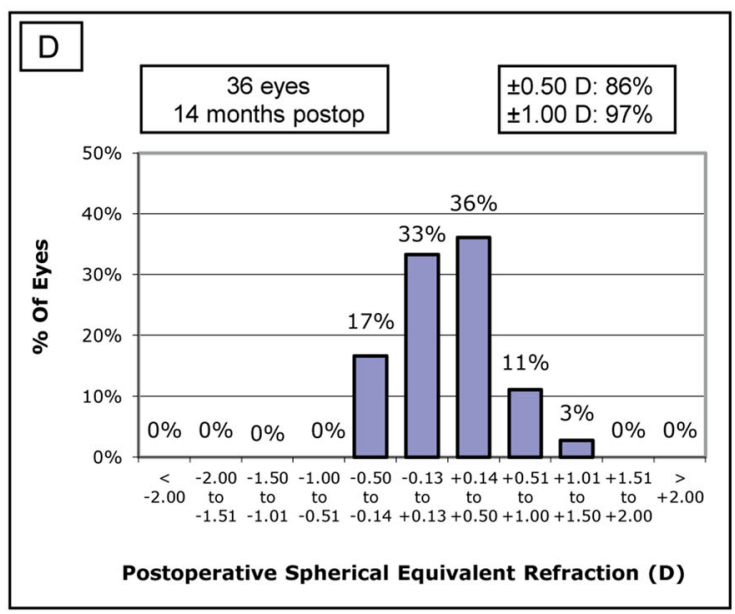

Spherical Equivalent Refractive Accuracy 
4 Fig. 1 Refractive outcomes for unilateral SMILE patients. Postoperative cumulative uncorrected distance visual acuity (UDVA) after small incision lenticule extraction (SMILE) (A). Postoperative changes in UDVA lines (B). Attempted vs. achieved spherical equivalent refraction (C). Accuracy of spherical equivalent refraction (D). Postoperative refractive astigmatism $(\mathbf{E})$

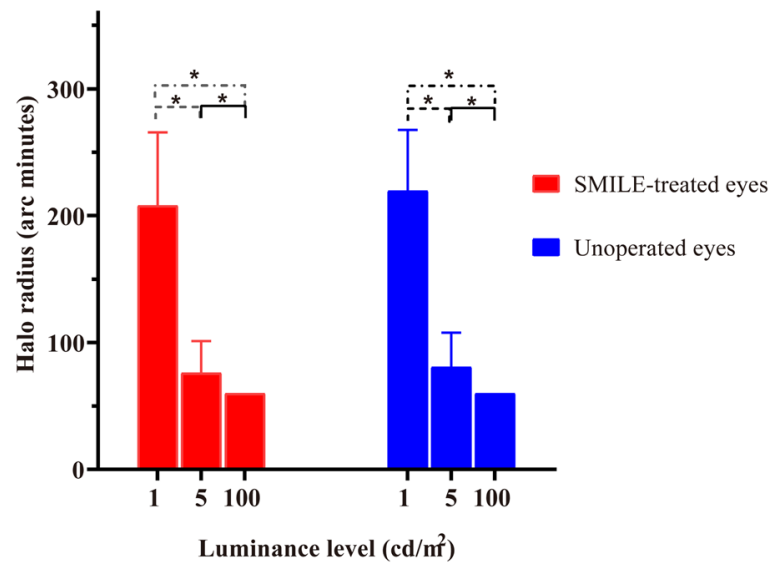

Fig. 2 Disk halo size at letter luminance levels of 1, 5, and $100 \mathrm{~cd} / \mathrm{m}^{2}$ in SMILE-treated and unoperated eyes of patients with myopic anisometropia

\section{Nighttime Symptoms and Satisfaction}

As Fig. 4 shows, none of the patients reported moderate or severe symptoms at night. Nineteen of the 36 patients reported no nighttime symptoms, while 13 patients reported mild symptoms (glare, halo, starburst) in both eyes. Among these, nine patients reported mild glare, two reported halo, ten reported starburst, and none reported distortion. No patients reported worse nighttime symptoms in the SMILE-treated eye compared to the unoperated eye, whereas four patients, who had interocular visual differences, reported better night vision in SMILE-treated eyes than in unoperated eyes (Table 4). It is noted that the unoperated eyes of case 4, who reported mild night vision disturbance, showed poor uncorrected contrast sensitivity at high spatial frequencies of 7.1 and 14.6 cycles per degree $(13 \mathrm{~dB}$ and $6 \mathrm{~dB}$, respectively), but the SMILE-treated eye was normal.

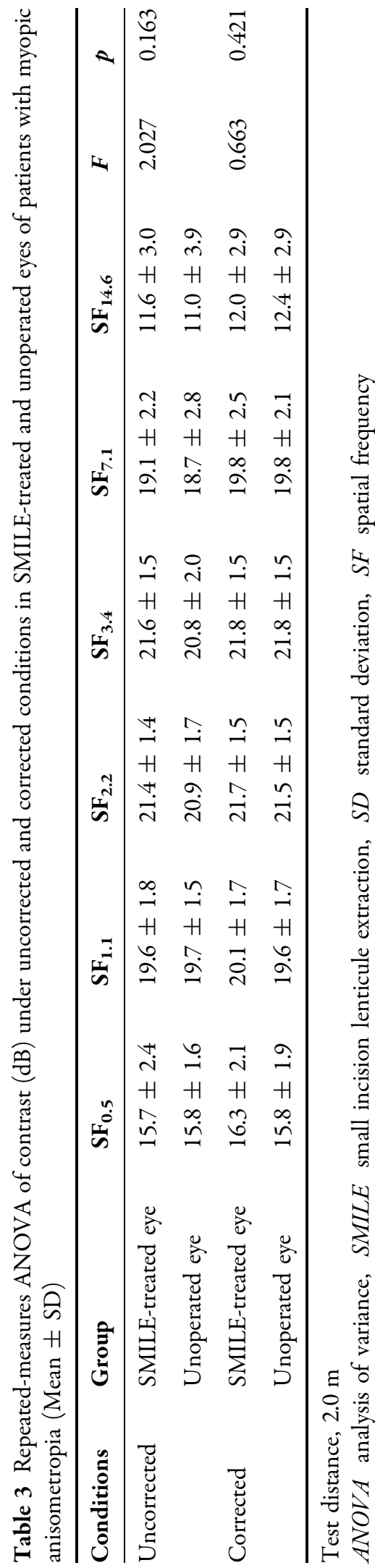




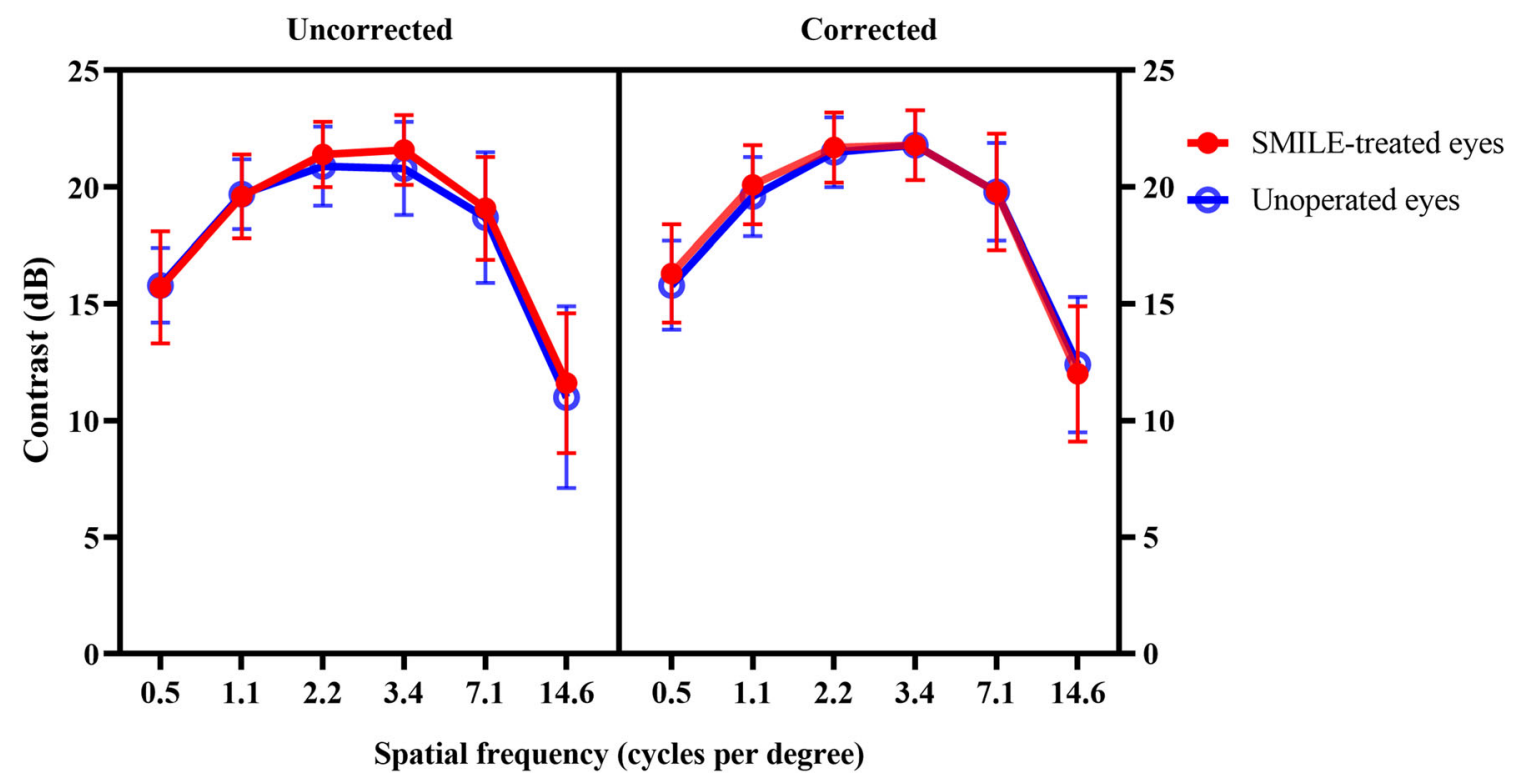

Fig. 3 Contrast at spatial frequencies of $0.5,1.1,2.2,3.4,7.1$, and 14.6 cycles per degree without (left) and with (right) spectacle correction between SMILE-treated and unoperated eyes

The mean satisfaction score of the 36 enrolled patients was $9.39 \pm 0.80$ (range, $8-10$ ).

\section{DISCUSSION}

Although many studies have documented visual outcomes and optical quality after SMILE, few studies have compared both eyes of the same person. Since nighttime symptoms such as glare or halo are subjective symptoms, the intraindividual contralateral real-time design is undoubtedly a strength of this study. In this study, we report similar visual performance between SMILE-treated and unoperated eyes for disk halo size, contrast sensitivity, and nighttime symptoms in patients with anisometropia who underwent monocular SMILE.

An efficacy index of 1.18 and safety index of 1.28 were obtained, which were in line with previously reported values $[2,6,9]$. Good visual acuity is the premise to discuss visual quality.

To simulate patients' routine experience, the halo radius was measured under mesopic and photopic $\left(1 \mathrm{~cd} / \mathrm{m}^{2}, 5 \mathrm{~cd} / \mathrm{m}^{2}, 100 \mathrm{~cd} / \mathrm{m}^{2}\right)$ vision. The halo radius in both eyes decreased with an increase in the letter luminance. The halo radius obtained at a letter luminance of $1 \mathrm{~cd} / \mathrm{m}^{2}$ was almost three times the value obtained at a higher luminance $\left(5 \mathrm{~cd} / \mathrm{m}^{2}\right)$, which was close to the values in this age range [11]. Furthermore, no differences were found between the two eyes of the same patients. This was in line with our previous study where disk halo size returned to the baseline value at 3 months postoperatively [13]. The limitation of that study was the lack of subjective survey and contrast sensitivity tests. In the present study, we remedied these issues. In the current study, $89 \%$ of patients reported similar symptoms in both eyes, and no patients reported moderate or severe symptoms at night. In addition, high satisfaction scores were observed. $\mathrm{Xu}$ and Yang [6] evaluated myopic patients using the Arnold questionnaire and found that $27 \%$ of the patients reported mild to moderate glare symptoms 2 weeks after SMILE, which eventually decreased to $2 \%$ at 1 year postoperatively. Gyldenkerne et al. [8] found that none of the 51 patients reported severe symptoms 3 months after surgery, and 20 patients had no nighttime symptoms. Although the questionnaires used in the present and previous studies were different, the final results showed agreement that the nighttime symptoms were able to return to normal in most of the patients. Moreover, similar to previous 


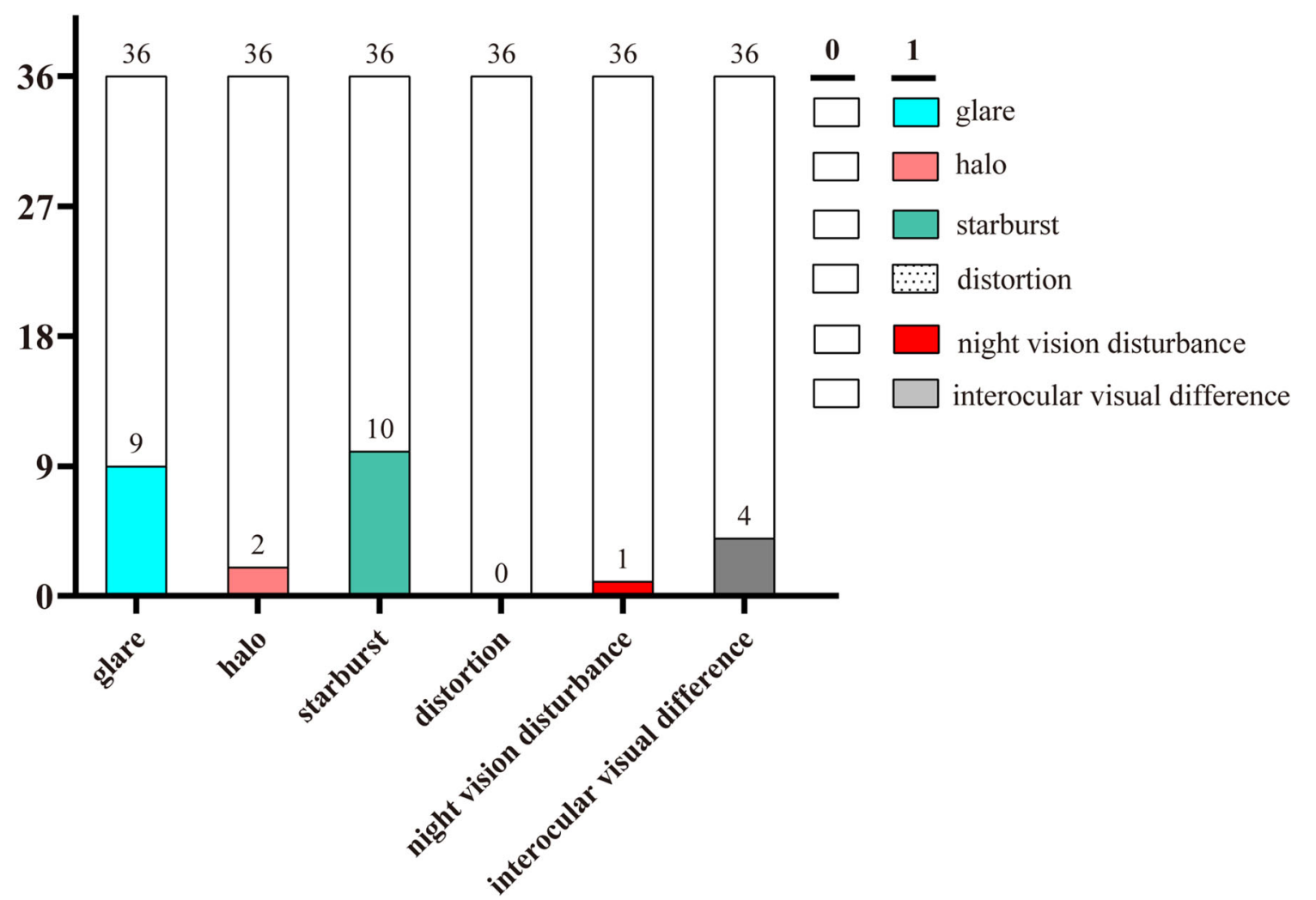

Fig. 4 The number of patients who reported nighttime symptoms: 17 of the 36 patients reported nighttime symptoms. Among them, 13 patients reported mild symptoms in both eyes, whereas four patients reported

studies $[5,8]$, glare and starburst were the most common night symptoms after SMILE.

Contrast sensitivity tests also showed no significant differences between SMILE-treated and unoperated eyes at all SFs under both corrected and uncorrected conditions. This is in agreement with previous studies. It has been reported that photopic and mesopic contrast sensitivity showed a sharp decline at first, and then recovered at 1-3 months postoperatively $[6,9,17]$. A decrease in contrast sensitivity in the early stage after SMILE might correlate with corneal backscatter when light passes through the corneal stroma $[18,19]$ and microdistortions in Bowman's layer [20], together with inflammatory responses [21]. In the first cohort of patients undergoing SMILE procedures in the world, Sekundo et al. [22] showed no significant changes in either mesopic or photopic contrast sensitivity at 3, 6, and 12 months symptoms in the unoperated eye. Symptom scores: 0 , none; 1 , mild; 2 , moderate; 3 , severe

postoperatively. Contrast sensitivity after the SMILE procedure was comparable to that in normal eyes.

Interestingly, the contralateral unoperated eye became more myopic, while the SMILEtreated eye was slightly overcorrected. The average SE of the unoperated eye ranged from $-0.08 \pm 0.66 \mathrm{D}$ to $-0.22 \pm 0.39 \mathrm{D}$. The slope of the attempted versus achieved SE correction was 1.0376. Hence, the unoperated eye became more myopic, as the accommodation always affects both eyes simultaneously. It might also be because $80.6 \%(29 / 36)$ of the cases were the dominant eye, and theoretically, dominant eyes perform better than non-dominant eyes. Shapira et al. [23] also observed overcorrection in the more myopic eyes in 472 eyes of 236 patients with anisometropia. This should be considered during monocular SMILE design. 
Table 4 Characteristics of patients reporting interocular visual difference

\begin{tabular}{llllllll}
\hline Cases & $\begin{array}{l}\text { Sex/age } \\
\text { (years) }\end{array}$ & Eye & Manifest refraction & $\begin{array}{l}\text { Axial length } \\
\text { elongation }(\mathbf{m m})\end{array}$ & $\begin{array}{l}\text { UDVA } \\
(\mathbf{l o g M A R})\end{array}$ & $\begin{array}{l}\text { Pupil size /OZ } \\
(\mathbf{m m})\end{array}$ & $\begin{array}{l}\text { Night } \\
\text { vision }\end{array}$ \\
\hline 1 & $\mathrm{~F} / 24$ & $\mathrm{SE}$ & $+0.75 \rightarrow 1.5$ & - & -0.2 & $6.2 / 6.7$ & Better \\
& & $\mathrm{UE}$ & $-0.25 /-0.25 \times 175 \rightarrow 1.2$ & 0.11 & 0.1 & $6.4 /-$ & - \\
2 & $\mathrm{M} / 19$ & $\mathrm{SE}$ & $+0.5 \rightarrow 1.2$ & - & -0.2 & $8.3 / 6.9$ & Better \\
& & $\mathrm{UE}$ & $-1.25 \rightarrow 1.2$ & 0.09 & 0.3 & $8.0 /-$ & - \\
3 & $\mathrm{~F} / 26$ & $\mathrm{SE}$ & $+0.25 /-0.25 \times 35 \rightarrow 1.5$ & - & -0.1 & $6.6 / 6.9$ & Better \\
& & $\mathrm{UE}-1.00 /-1.00 \times 175 \rightarrow 1.0$ & 0.19 & 0.3 & $6.8 /-$ & - \\
4 & $\mathrm{~F} / 19$ & $\mathrm{SE}$ & $+0.25 \rightarrow 1.5$ & - & -0.2 & $8.3 / 6.7$ & Better \\
& & $\mathrm{UE}$ & $-0.50 /-0.50 \times 100 \rightarrow 1.2$ & 0.02 & 0.2 & $8.2 /-$ & - \\
\hline
\end{tabular}

SE, SMILE-treated eye; UE, unoperated eye; OZ, optical zone

Our study has some limitations. Whether anisometropia plays a relevant role in nighttime symptoms is unclear. However, as mentioned before, the contralateral real-time design has its own advantage in real-time comparison. Moreover, as shown in Table 4, the postoperative difference in SE might skew the data in favor of the SMILE-operated eye. Additionally, the preoperative $\mathrm{SE}$ of the SMILE-treated eye was $-3.77 \pm 1.56 \mathrm{D}$, and the optical zone was larger than $6.5 \mathrm{~mm}$; therefore, a study of high myopic anisometropia with a small optical zone is needed to verify the present outcomes.

\section{CONCLUSIONS}

In conclusion, this study showed similar contrast sensitivity between SMILE-treated and unoperated eyes after SMILE, and almost normal nighttime values were achieved after SMILE.

\section{ACKNOWLEDGEMENTS}

We thank the participants of the study, and the Ethics Committee of the EENT Hospital of Fudan University. A consent form was obtained from each patient.
Funding. This study, including the Journal's Rapid Service Fee, was partly supported by the Shanghai Sailing Program (Grant No. 20YF1405000), the National Natural Science Foundation of China for Young Scholars (Grant No. 82000929), the National Natural Science Foundation of China (Grant No. 81770955), Joint Research Project of New Frontier Technology in Municipal Hospitals (SHDC12018103), Shanghai Science and Technology Project (Grant No. 20410710100), Major Clinical Research Project of Shanghai Shenkang Hospital Development Center (SHDC2020CR1043B), and Project of Shanghai Xuhui District Science and Technology (2020015).

Authorship. All authors meet the International Committee of Medical Journal Editors (ICMJE) criteria for authorship for this article, take responsibility for the integrity of the work as a whole, and have given their approval for this version to be published.

Authorship Contributions. Study concept and design (Wuxiao Zhao, Tian Han, Xingtao Zhou), data collection (Wuxiao Zhao, Tian Han, Aruma Aruma), analysis and interpretation of data (Wuxiao Zhao, Tian Han, Meiyan Li, Walter Sekundo, Xingtao Zhou), writing the manuscript (Wuxiao Zhao, Tian Han, Meiyan Li, Aruma Aruma), critical revision of the 
manuscript (Wuxiao Zhao, Tian Han, Walter Sekundo, Xingtao Zhou), Obtaining funding and supervision (Xingtao Zhou).

Disclosures. Wuxiao Zhao, Tian Han, Meiyan Li, Walter Sekundo, Aruma Aruma and Xingtao Zhou confirm that they have nothing to declare. The authors declare that the research was conducted in the absence of any commercial or financial relationships that could be construed as a potential conflict of interest.

Compliance with Ethics Guidelines. Our study was approved by the ethics committee of the Eye \& ENT Hospital of Fudan University and performed in accordance with the Helsinki Declaration of 1964 and its later amendments. Please find the attached original text (reference numbers: KJ2010-18).

A consent form for participation and academic publication request was obtained from each patient prior to the study.

Data Availability. All data generated or analyzed during this study are included in this published article/as supplementary information files.

Open Access. This article is licensed under a Creative Commons Attribution-NonCommercial 4.0 International License, which permits any non-commercial use, sharing, adaptation, distribution and reproduction in any medium or format, as long as you give appropriate credit to the original author(s) and the source, provide a link to the Creative Commons licence, and indicate if changes were made. The images or other third party material in this article are included in the article's Creative Commons licence, unless indicated otherwise in a credit line to the material. If material is not included in the article's Creative Commons licence and your intended use is not permitted by statutory regulation or exceeds the permitted use, you will need to obtain permission directly from the copyright holder. To view a copy of this licence, visit http://creativecommons.org/licenses/by$\mathrm{nc} / 4.0 /$.

\section{REFERENCES}

1. Blum M, Lauer AS, Kunert KS, Sekundo W. 10-year results of small incision lenticule extraction. J Refract Surg. 2019;35(10):618-23.

2. Han T, Xu Y, Han X, Zeng L, Shang J, Chen X, et al. Three-year outcomes of small incision lenticule extraction (SMILE) and femtosecond laser-assisted laser in situ keratomileusis (FS-LASIK) for myopia and myopic astigmatism. $\mathrm{Br} \mathrm{J}$ Ophthalmol. 2019;103(4):565-8.

3. Miao H, He L, Shen Y, Li M, Yu Y, Zhou X. Optical quality and intraocular scattering after femtosecond laser small incision lenticule extraction. J Refract Surg. 2014;30(5):296-302.

4. Xu L, Wang Y, Li J, Liu Y, Wu W, Zhang H, et al. Comparison of forward light scatter changes between SMILE, femtosecond laser-assisted LASIK, and Epipolis LASIK: results of a 1-year prospective study. J Refract Surg. 2015;31(11):752-8.

5. Han T, Xu Y, Han X, Shang J, Zeng L, Zhou X. Quality of life impact of refractive correction (QIRC) results three years after SMILE and FS-LASIK. Health Qual Life Outcomes. 2020;18(1):107.

6. Xu Y, Yang Y. Small-incision lenticule extraction for myopia: results of a 12-month prospective study. Optom Vis Sci. 2015;92(1):123-31.

7. Chiche A, Trinh L, Saada O, Faure JF, Auclin F, Baudouin C, et al. Early recovery of quality of vision and optical performance after refractive surgery: small-incision lenticule extraction versus laser in situ keratomileusis. J Cataract Refract Surg. 2018;44(9):1073-9.

8. Gyldenkerne A, Ivarsen A, Hjortdal J. Optical and visual quality after small-incision lenticule extraction. J Cataract Refract Surg. 2019;45(1):54-61.

9. Ang M, Farook M, Htoon HM, Tan D, Mehta JS. Simulated night vision after small-incision lenticule extraction. J Cataract Refract Surg. 2016;42(8): 1173-80.

10. Mainster MA, Turner PL. Glare's causes, consequences, and clinical challenges after a century of ophthalmic study. Am J Ophthalmol. 2012;153(4): 587-93.

11. Puell MC, Perez-Carrasco MJ, Barrio A, Antona B, Palomo-Alvarez C. Normal values for the size of a halo produced by a glare source. J Refract Surg. 2013;29(9):618-22.

12. Puell MC, Perez-Carrasco MJ, Palomo-Alvarez C, Antona B, Barrio A. Relationship between halo size 
and forward light scatter. $\mathrm{Br} \mathrm{J}$ Ophthalmol. 2014;98(10):1389-92.

13. Han T, Zhao F, Chen X, Miao H, Chen Z, Zhou X. Evaluation of disk halo size after small incision lenticule extraction (SMILE). Graefes Arch Clin Exp Ophthalmol. 2019;257(12):2789-93.

14. Damgaard IB, Ang M, Farook M, Htoon HM, Mehta JS. Intraoperative patient experience and postoperative visual quality after SMILE and LASIK in a randomized, paired-eye, controlled Study. J Refract Surg. 2018;34(2):92-9.

15. Chen X, Han T, Zhao F, Miao H, Wang X, Zhou X. Evaluation of Disk Halo Size after implantation of a collamer lens with a central hole (ICL V4c). J Ophthalmol. 2019;2019:7174913.

16. Asgari S, Hashemi H, Mohamadi A, Jafarzadehpur E, Miraftab M, Shahhoseini S, et al. Scotopic contrast sensitivity and glare after accelerated corneal crosslinking. Clin Exp Optom. 2018;101(1):52-6.

17. Liu M, Chen Y, Wang D, Zhou Y, Zhang X, He J, et al. Clinical outcomes After SMILE and femtosecond laser-assisted LASIK for myopia and myopic astigmatism: a prospective randomized comparative study. Cornea. 2016;35(2):210-6.

18. Han T, Zhao J, Shen Y, Chen Y, Tian M, Zhou X. A Three-year observation of corneal backscatter after small incision lenticule extraction (SMILE). J Refract Surg. 2017;33(6):377-82.

19. Agca A, Ozgurhan EB, Yildirim Y, Cankaya KI, Guleryuz NB, Alkin Z, et al. Corneal backscatter analysis by in vivo confocal microscopy: fellow eye comparison of small incision lenticule extraction and femtosecond laser-assisted LASIK. J Ophthalmol. 2014;2014:265012.

20. Yao P, Zhao J, Li M, Shen Y, Dong Z, Zhou X. Microdistortions in Bowman's layer following femtosecond laser small incision lenticule extraction observed by Fourier-Domain OCT. J Refract Surg. 2013;29(10):668-74.

21. Dong Z, Zhou X, Wu J, Zhang Z, Li T, Zhou Z, et al. Small incision lenticule extraction (SMILE) and femtosecond laser LASIK: comparison of corneal wound healing and inflammation. Br J Ophthalmol. 2014;98(2):263-9.

22. Sekundo W, Gertnere J, Bertelmann T, Solomatin I. One-year refractive results, contrast sensitivity, high-order aberrations and complications after myopic small-incision lenticule extraction (ReLEx SMILE). Graefes Arch Clin Exp Ophthalmol. 2014;252(5):837-43.

23. Shapira Y, Vainer I, Mimouni M, Levartovsky S, Sela T, Munzer G, et al. Effect of anisometropia on the predictability and accuracy of refractive surgery. Cornea. 2016;35(11):1410-5. 